\title{
Enhanced Seq2Seq Autoencoder via Contrastive Learning for Abstractive Text Summarization
}

\author{
Chujie Zheng ${ }^{1, *}$, Kunpeng Zhang ${ }^{2}$, Harry Jiannan Wang ${ }^{1}$, Ling Fan ${ }^{3,4}$, Zhe Wang ${ }^{4}$ \\ ${ }^{1}$ University of Delaware \\ ${ }^{2}$ University of Maryland, College Park \\ ${ }^{3}$ Tongji University, ${ }^{4}$ Tezign \\ chz@udel .edu, kpzhang@umd.edu, hjwang@udel .edu, \\ lfan@tongji.edu.cn, wetezign.com
}

\begin{abstract}
In this paper, we present a denoising sequenceto-sequence (seq2seq) autoencoder via contrastive learning for abstractive text summarization. Our model adopts a standard Transformer-based architecture with a multilayer bi-directional encoder and an autoregressive decoder. To enhance its denoising ability, we incorporate self-supervised contrastive learning along with various sentencelevel document augmentation. These two components, seq2seq autoencoder and contrastive learning, are jointly trained through fine-tuning, which improves the performance of text summarization with regard to ROUGE scores and human evaluation. We conduct experiments on two datasets and demonstrate that our model outperforms many existing benchmarks and even achieves comparable performance to the state-of-the-art abstractive systems trained with more complex architecture and extensive computation resources.
\end{abstract}

\section{Introduction}

Text summarization aims to produce an accurate text snippet to capture the key information. Existing methods are either extractive or abstractive. Extractive methods select sentences from the document and the abstractive methods generate sentences based on the input document as a summary. With the advancement of natural language processing (NLP) research, especially in the area of largescale pre-trained language models (Devlin et al., 2019; Peters et al., 2018; Radford et al., 2019; Liu et al., 2019) in recent years, abstractive summarization has become a popular research topic and made significant progress. In most of existing abstractive summarization models, such as BART (Lewis et al., 2020b), PEGASUS (Zhang et al., 2020) and ProphetNet (Qi et al., 2020), all adopt Transformerbased architecture (Vaswani et al., 2017). They are usually first pre-trained in an unsupervised manner with a large amount of corpus and then fine-tuned

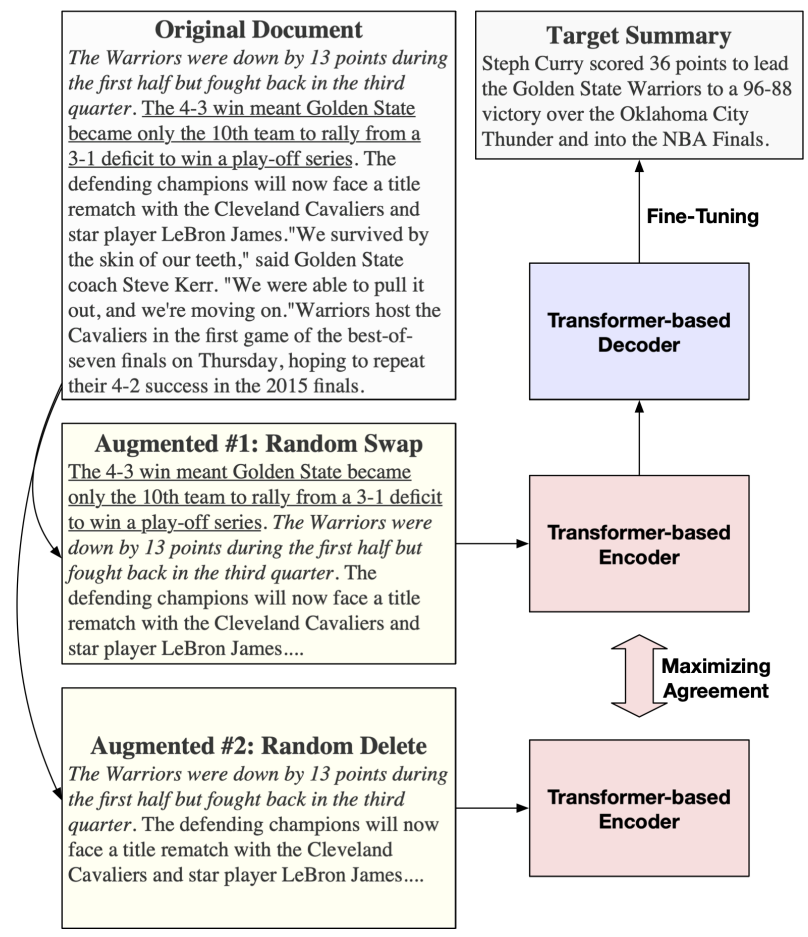

Figure 1: An illustration example of ESACL.

on a specific dataset for supervised downstream applications. These models have shown superiority on various text understanding tasks, especially for generating abstractive summaries.

Despite impressive performance on standard benchmarks, these deep networks are often brittle when deployed in real-world systems (Goel et al., 2021). The primary reason lies in that they are not robust to various noises, such as data corruption (Belinkov and Bisk, 2018), distribution shift (Hendrycks et al., 2020) or harmful data manipulation (Jia and Liang, 2017). In addition, they may also heavily rely on spurious patterns for prediction (McCoy et al., 2019). As demonstrated in prior studies, the seq2seq model plays a critical role in many downstream applications. Thus, we expect to enable its denoising capability when developing such a seq2seq model in NLP tasks. 
Furthermore, many prior studies in language understanding find that the global semantics may significantly be neglected by Transformer-based models (Fang and Xie, 2020). Because self-attention in these models is usually applied to learn and predict word-level characteristics during pre-training. The sentence embeddings aggregated from word embeddings learned by existing pre-trained language models have been found not be able to effectively and sufficiently capture the semantics among sentences ( $\mathrm{Li}$ et al., 2020a). This can lead to poor performance for subsequent tasks, e.g., abstractive summarization. The reason is that summarization requires wide-coverage natural language understanding going beyond the meaning of individual words and sentences (Liu and Lapata, 2019). Therefore, to build a denoising seq 2 seq model, state-of-the-art (SOTA) approaches like BART (Lewis et al., 2020b) and MARGE (Lewis et al., 2020a) developed new objectives for pretraining. BART is trained by first corrupting documents at a word level and then optimizing a reconstruction loss between the generated output and the original document. MARGE learns the model by self-supervising the reconstruction of target text where it first retrieves a set of related texts and then maximize the likelihood of generating the original documents based on selected texts. All these seq2seq-based approaches are inspirational and emphasize the ability of denoising and modeling global semantics.

In this study, we propose a new framework ESACL, Enhanced Seq2Seq Autoencoder via Contrastive Learning, to improve the denoising ability of the seq2seq model and increase the model flexibility by achieving our goal through finetuning. Unlike most existing methods that design denoising objectives in pre-training, ESACL optimizes the model in the fine-tuning phase which requires less computation resources and significantly saves training time. Specifically, ESACL leverages self-supervised contrastive learning (Chen et al., 2020; He et al., 2020) and integrates it into a standard seq2seq autoencoder framework. Overall, it involves two stages: (1) sentence-level document augmentation, and (2) joint learning framework of seq2seq autoencoder and contrastive learning with an overall objective based on a fine-tuning loss and a self-supervised contrastive loss. Regarding the seq2seq autoencoder, ESACL uses a similar architecture to BART, which is a standard transformer- based model with a multi-layer bi-directional encoder and left-to-right decoder. As shown in Figure 1, ESACL performs document augmentation to create two instances, and designs a unique framework underlying the seq2seq model: it not only uses the output from the decoder for fine-tuning but also tries to maximize agreement of the output from the encoder between two augmented instances.

A key step in contrastive learning is data augmentation. Various augmentation strategies have been developed in many NLP tasks at the word level, such as inserting a new word or swapping two tokens. To capture high-level semantics and the structural information of the entire document, we perform data augmentation at the sentence level. In this study, we implement several combinations of data augmentation and our experiment results show that (i) the model performance can be improved with sentence-level augmentation; (ii) the summarization performance with different data augmentation strategies does not vary much; (iii) the augmentation that largely interrupts the structure of the document should be avoided.

To sum up, ESACL proposes a new way of denoising a seq2seq model via fine-tuning for abstractive summarization. It presents a new scheme for summarization which incorporates self-supervised contrastive learning into a seq2seq framework to improve the model flexibility. The major contributions of this study are as follows:

- We propose ESACL, a new abstractive text summarization framework that jointly trains a seq2seq autoencoder with contrastive learning through fine-tuning.

- We evaluate ESACL using two summarization datasets through quantitative measurement, robustness check, and human evaluation. ESACL achieves state-of-the-art performance and has shown better flexibility concerning modeling potential irrelevant noises.

- We introduce several sentence-level document augmentation strategies and conduct an ablation study to understand their impact on the performance.

\section{Related Work}

Three lines of research are closely related to our paper: abstractive text summarization, contrastive learning, and data augmentation.

Abstractive text summarization has achieved promising results with the rapid development of 


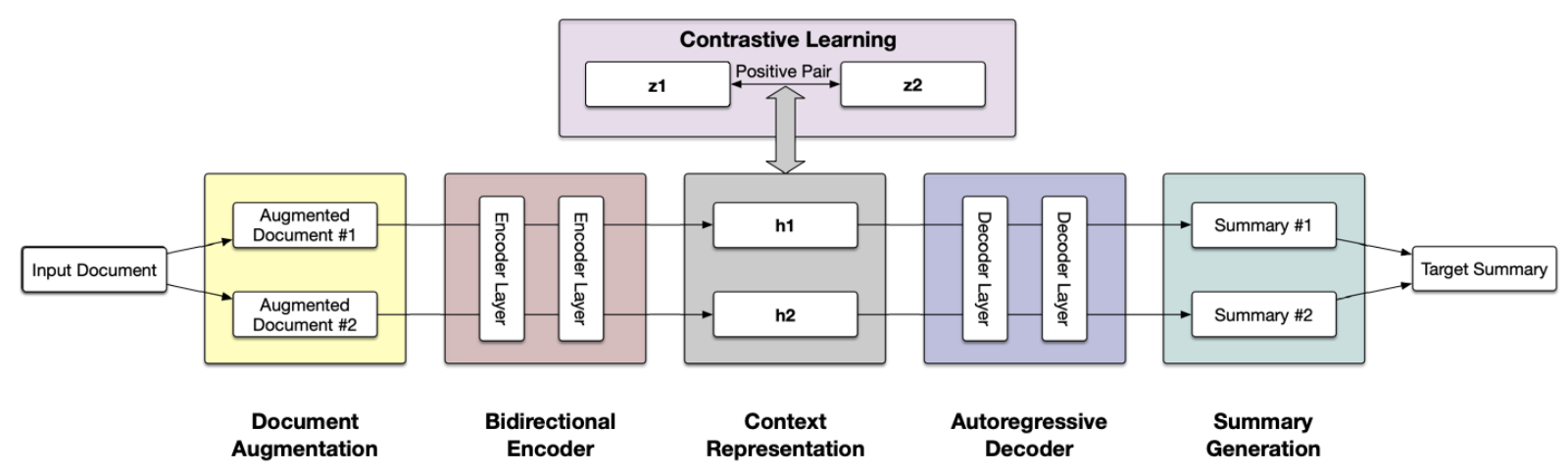

Figure 2: The overall architecture of our proposed ESACL.

deep learning. Neural network-based models (Rush et al., 2015; Nallapati et al., 2016; Chopra et al., 2016; Nallapati et al., 2017; Zhou et al., 2017; Tan et al., 2017; Gehrmann et al., 2018; Zhu et al., 2019) enable the framework for generating abstractive summary. Recently, with the success of attention mechanism and Transformer-based (Vaswani et al., 2017) language models, pre-training based methods (Devlin et al., 2019; Radford et al., 2019) have attracted growing attention and achieved stateof-the-art performances in many NLP tasks, and pre-training encoder-decoder Transformers (Song et al., 2019; Lewis et al., 2020b; Zhang et al., 2020; Qi et al., 2020; Lewis et al., 2020a) show great successes for the summarization.

Contrastive learning has been recently a resurgence in image analysis and language understanding (Khosla et al., 2020; Chen et al., 2020; Fang and Xie, 2020; Gunel et al., 2020). Researchers have developed many contrastive learning-based frameworks, including self-supervised framework (Fang and Xie, 2020) and supervised framework (Gunel et al., 2020) and apply them to different language understanding tasks, e.g., sentiment analysis (Li et al., 2020b) and document clustering (Shi et al., 2020). They mainly use contrastive learning to help models deeply explore the unique characteristics of data while ignoring irrelevant noises, which also motivates the present study.

Data augmentation is the key in contrastive learning and has been widely applied in image analysis (Wong et al., 2016). Textual data augmentation is different and can be mainly categorized into word-level transformation (Kolomiyets et al., 2011; Wang and Yang, 2015; Zhang et al., 2015; Qiu et al., 2020) and neural text generation (Sennrich et al., 2016; Yu et al., 2018). In our paper, to preserve the global semantics while filtering irrelevant noise for a document, we design several sentence-level augmentation strategies and show their effectiveness in summarization. Based on the experiment results, we believe that developing new alternative augmentations for text summarization has its great merit.

\section{Preliminary}

Automatic text summarization aims at condensing a document to a shorter version while preserving the key information. Let $\mathbf{d}=\left\{\mathbf{x}_{1}, \mathbf{x}_{2}, \ldots, \mathbf{x}_{N}\right\}$ be an input document with $N$ tokens and $\mathbf{x}_{i}$ is the word embedding for the $i$-th token. Given a document $\mathbf{d}$, we expect to learn a function $f(\mathbf{d})$ that maps $\mathbf{d}$ to another sequence of tokens $\mathbf{y}=\left\{\mathbf{y}_{1}, \mathbf{y}_{2}, \cdots, \mathbf{y}_{m}\right\}$, where $\mathbf{y}$ is the generated summary with $m$ tokens. $m$ is an unknown apriori and depends on the input sequence and the task-specific requirement.

Such a function $f(\cdot)$ is often implemented by a seq2seq model. The key idea is to represent an input sequence as a low-dimensional vector while preserving the contextual information in the sequence as much as possible, upon which a new task-specific sequence with an arbitrary length can be automatically generated (Jurafsky and Martin, 2020). A typical seq2seq model usually consists of three components:

- An encoder, denoted as $f_{\text {encoder }}$ that accepts an input sequence $\mathbf{d}$, and generates a corresponding sequence of contextualized representation $\mathbf{h}$.

- A context vector, $\mathbf{c}$ that is a function of $\mathbf{h}$ and conveys the essence of the input to the decoder.

- And a decoder, $f_{\text {decoder }}$ that uses $\mathbf{c}$ to generate an arbitrary length of sequence $\mathbf{y}$ based on the task-specific requirement.

\section{Our Proposed Model}

In this section, we present our proposed model ESACL, which leverages self-supervised con- 
trastive learning to enhance the denoising ability of a seq2seq framework. Figure 2 illustrates the overall architecture of ESACL. For a given input document d, ESACL first creates a pair of augmented documents that are expected to associate with the same original target summary. ESACL then generates the latent representation of the augmented documents using the Transformer-based encoder and performs self-supervised contrastive learning to encourage the model to capture potential noises in the document $\mathbf{d}$. Finally the optimized latent representation is sent to the Transformer-based decoder to generate the summary. In Section 4.2, we present our implementation of contrastive learning in ESACL. In Section 4.1, we introduce several sentence-level document augmentation strategies which are the key in contrastive learning. In Section 4.3, we describe the detailed seq2seq architecture of ESACL, in particular how the self-supervised contrastive learning is incorporated and how they are jointly trained via fine-tuning.

\subsection{Document Augmentation}

Data augmentation has been used to increase the denoising capability of a model. In prior literature as mentioned in Section 2, there exists many contrastive learning-based models and applications in NLP. However, most of these methods focus on augmentation at the word level, which might not be suitable for text summarization because the global semantics and even noises at a higher level (e.g., sentence or document) can be easily ignored. In this study, we perform document augmentation at the sentence level. Specifically, given an input document $\mathbf{d}$ with a sequence of $k$ sentences, we manipulate the document via various transformations at the sentence level to augment the document. By doing this, ESACL can generate another sequence $\hat{\mathbf{d}}$ where main semantics are preserved with some additional noises.

Similar to Qiu et al. (2020), we design several document augmentation approaches at the sentence level, as follows:

- Random Insertion (RI): randomly pick an existing sentence and insert it into a random position in the input document.

- Random Swap (RS): randomly select two sentences and swap their positions.

- Random Deletion (RD): randomly delete a sentence from the input document.

- Document Rotation (DR): randomly select a sentence and the document is rotated using this selected sentence as the pivot.

\subsection{Self-Supervised Contrastive Learning}

We introduce self-supervised contrastive learning into ESACL during the fine-tuning process to enhance its noising flexibility. ESACL performs document augmentation of the original input document to create positive training pairs. Along with negative pairs (two different documents), ESACL is able to encourage itself to identify if two context vectors learned from the encoder are representing the same original input document. By doing so, ESACL improves the quality of the context vector $\mathbf{c}$ during the fine-tuning which can benefit the performance of downstream language generation.

To form positive pairs during training, we perform document augmentation to create two augmented instances for each document in a batch of $K$ training instances $b=\left\{\mathbf{d}_{1}, \mathbf{d}_{2}, \ldots, \mathbf{d}_{K}\right\}$. Suppose $\mathbf{d}_{i}$ is the original input document, we generate the augmented documents $\hat{\mathbf{d}}_{2 i-1}=A_{1}\left(\mathbf{d}_{i}\right)$ and $\hat{\mathbf{d}}_{2 i}=A_{2}\left(\mathbf{d}_{i}\right)$, where $A$ refers to a specific augmentation strategy. Thus, we have $2 K$ augmented instances in total for a batch, and we assume $\hat{\mathbf{d}}_{2 i-1}$ and $\hat{\mathbf{d}}_{2 i}$ are augmented from the same input document $\mathbf{d}_{i}$. A positive pair is defined if and only if two instances are from the same original input document. Otherwise they are considered as a negative pair. We use the pre-trained encoder $f_{\text {encoder }}(\cdot)$ to obtain the latent representation of each augmented document $\hat{\mathbf{d}}$ as $\mathbf{h}=f_{\text {encoder }}(\hat{\mathbf{d}})$. In our work, we use the final hidden vector corresponding to the first input token as the aggregate representation for the document like prior literature did (Devlin et al., 2019). ESACL also applies a non-linear projection head $g$ to further understand the deep semantics among latent dimensions. It projects the representation $\mathbf{h}$ into another latent space $\mathbf{z}=g(\mathbf{h})$, which is used to calculate the contrastive loss $l(i, j)$ for the positive pair as Equation 1. Here $\mathbb{1}_{[k \neq i]}$ is 1 when $k \neq i$ and 0 otherwise. $\tau$ is a temperature parameter. $\operatorname{sim}(\cdot, \cdot)$ is a cosine similarity measure.

$$
l(i, j)=-\log \frac{\exp \left(\operatorname{sim}\left(\mathbf{z}_{i}, \mathbf{z}_{j}\right) / \tau\right)}{\sum_{k=1}^{2 K} \mathbb{1}_{[k \neq i]} \exp \left(\operatorname{sim}\left(\mathbf{z}_{i}, \mathbf{z}_{k}\right) / \tau\right)}
$$

The loss of contrastive learning in ESACL is:

$$
\mathcal{L}_{\mathrm{cl}}=\frac{1}{2 K} \sum_{i=1}^{K}[l(2 i-1,2 i)+l(2 i, 2 i-1)]
$$




\subsection{Sequence-to-sequence Architecture}

For the abstractive text summarization, we follow the literature and adopt the Transformer-based seq2seq model, which has proven to be effective (see Section 2). A natural question arising here is how to leverage the denoising ability of contrastive learning in the seq2seq framework to improve the summarization. To answer this question, we design a combined loss to jointly learn model parameters. For each instance $\mathbf{d}_{i}$, we obtain two augmented instances: $\hat{\mathbf{d}}_{2 i-1}$ and $\hat{\mathbf{d}}_{2 i}{ }^{1}$, which are considered as a positive pair for self-supervised contrastive learning. They are also used to generate summaries $\hat{\mathbf{y}}_{2 i-1}$ and $\hat{\mathbf{y}}_{2 i}$. The generated summary is compared with the target summary of the original input document for calculating the fine-tuning loss, $\mathcal{L}_{\text {generate, }}$ which measures the generation performance. In this study, we define the $\mathcal{L}_{\text {generate }}$ as the cross-entropy loss. We also use the generated positive pair to calculate the contrastive learning loss as we introduced, which measures the noising flexibility of our model. Equation 3 summarizes the overall loss of ESACL as the weighted sum of two losses. A hyper-parameter $\alpha \in[0,1]$ is used to balance the importance of contrastive learning and the summary generation. The overall process of ESACL is summarized in Algorithm 1.

$$
\mathcal{L}=\alpha \mathcal{L}_{\mathrm{cl}}+(1-\alpha) \mathcal{L}_{\text {generate }}
$$

\section{Experiments}

\subsection{Experiment Setting}

We evaluate our model using two popular summarization datasets: the CNN/Daily Mail dataset (CNN/DM) (Hermann et al., 2015) and the extreme summarization dataset (XSUM) (Narayan et al., 2018). Our experiments are conducted with 3 NVIDIA V100 GPUs. We adopt a 12-layer encoder and a 6-layer decoder with 16 attention heads. We warm-start the model parameter with the distilBART pre-trained model ${ }^{2}$ and trains 5 epochs with a batch size of $16^{3}$. For projection head in contrastive learning, we implement a 2-layer MLP to project the representation to a 128-dimensional latent space. We use Adam optimizer with a learning

\footnotetext{
${ }^{1}$ Different augmentation strategies can be combined. For example, $\hat{\mathbf{d}}_{2 i-1}$ is augmented via RI while $\hat{\mathbf{d}}_{2 i}$ is via RS.

${ }^{2}$ We choose distil-BART provided by HuggingFace. For CNN/DM, we use "sshleifer/distilbart-cnn-12-6". For XSUM, we use "sshleifer/distilbart-xsum-12-6". Appendix A records the detailed implementation.

${ }^{3}$ It takes about 35 hours for 5 epochs on our machine.
}

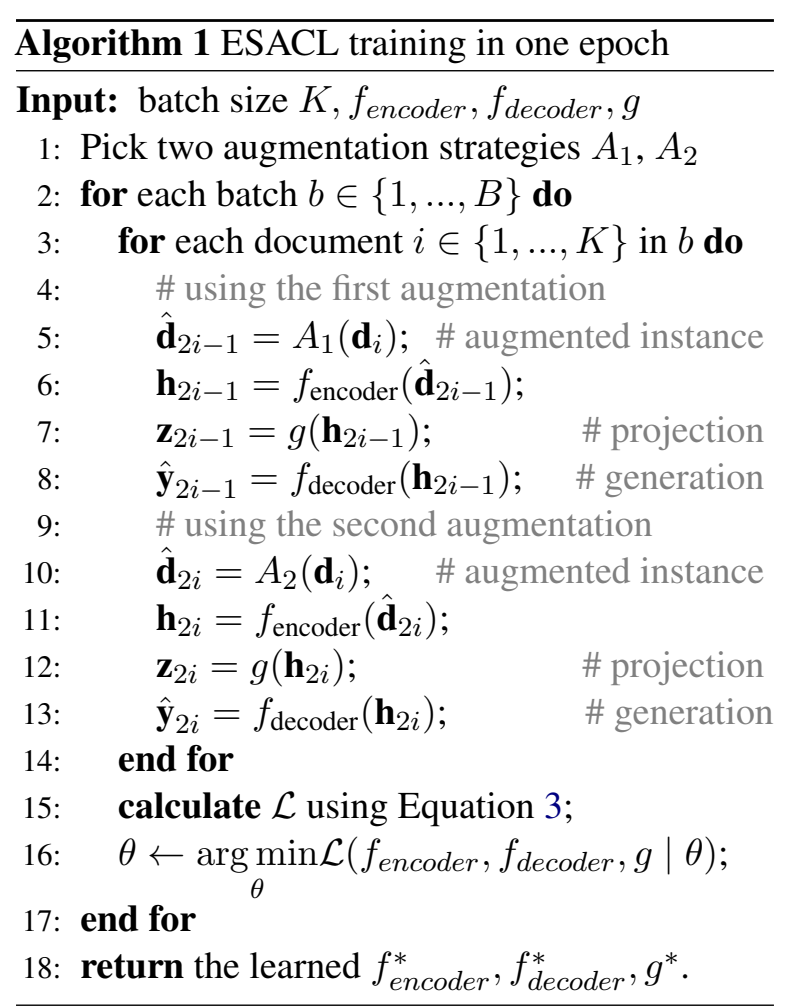

rate of $5 e-7$.

Given the limited computing resource (e.g., the memory limitation), we need to freeze some layers of the encoder to reduce the number of parameters. The impact of freezing different layers of the encoder will be discussed in the following ablation study (see Section 6.3). All results reported below are based on freezing the first 6 layers of the encoder. For the loss calculation, we set $\alpha=0.2$ and $\tau=0.5$. For data augmentation, we choose two augmentation operations, and discuss this hyperparameter in Section 6.3. For the purpose of reproducibility, all codes are publicly available here ${ }^{4}$.

\subsection{Experimental Results}

We compare our proposed model with the following cutting-edge summarization models.

- Lead-N uses the first $N$ sentences of the article as its summary.

- BERTSUM (Liu and Lapata, 2019) proposes a novel document-level encoder based on BERT to generate summary.

- MATCHSUM (Zhong et al., 2020) is an extractive summarization approach which formulates the task as a semantic text matching problem.

- PGNet (See et al., 2017) is the pointer-generator network, which copies words from the source text and retains the ability to produce novel words.

\footnotetext{
${ }^{4}$ https://github.com/chz816/esacl
} 
PGNet+Cov is with the coverage mechanism.

- BART (Lewis et al., 2020b) employs the bidirectional encoder to enhance the sequence understanding and the left-to-right decoder to generate the summary.

- PEGASUS (Zhang et al., 2020) introduces a new pre-train objective to encourage the model generate target sentences, which enables the model to capture global information among sentences.

- ProphetNet (Qi et al., 2020) predicts the next $n$ tokens simultaneously based on previous context tokens at each time step.

We adopt ROUGE (Lin, 2004) F1 score as the evaluation metric. We choose ROUGE-1, ROUGE2, and ROUGE-L for performance measurement, which are the common choices in the literature. We report the performance for all baseline models using the numbers from the original literature.

Results on CNN/DM: Table 1 records the performance on CNN/DM. ESACL outperforms most of the baseline models and achieves the highest ROUGE-L score on CNN/DM. Comparing to the SOTA extractive system MATCHSUM, ESACL achieves a higher ROUGE-2 and ROUGE-L score. Comparing to three SOTA abstractive systems, ESACL outperforms ProphetNet and improves the performance of BART by $7.3 \%$ on ROUGE-L. Our model achieves comparable performance with PEGASUS, which is the best-performed SOTA model.

\begin{tabular}{lccc}
\hline \multicolumn{1}{c}{ Model } & RG-1 & RG-2 & RG-L \\
\hline Lead-3 & 40.07 & 17.68 & 36.33 \\
\hline BERTSUM & 42.13 & 19.60 & 39.18 \\
MATCHSUM & $\mathbf{4 4 . 4 1}$ & 20.86 & 40.55 \\
\hline PGNet & 36.44 & 15.66 & 33.42 \\
PGNet+Cov & 39.53 & 17.28 & 36.38 \\
BART & 44.16 & 21.28 & 40.90 \\
ProphetNet & 43.68 & 20.64 & 40.72 \\
PEGASUS & 44.17 & $\mathbf{2 1 . 4 7}$ & $\mathbf{4 1 . 1 1}$ \\
\hline ESACL & $\mathbf{4 4 . 2 4}$ & $\mathbf{2 1 . 0 6}$ & $\mathbf{4 1 . 2 0}$ \\
\hline
\end{tabular}

Table 1: ROUGE (RG) evaluation on CNN/DM dataset

Results on XSUM: Table 2 records the ROUGE score on XSUM. ESACL outperforms the natural baseline and extractive systems. Our model achieves comparable performance to BART, and it is lower than the best-performed model PEGASUS.

The experimental results on two datasets show the effectiveness of the joint learning framework with contrastive learning, as indicated by the superior performance improvement of ESACL over

\begin{tabular}{lccc}
\hline \multicolumn{1}{c}{ Model } & RG-1 & RG-2 & RG-L \\
\hline Lead-1 & 16.30 & 1.60 & 11.95 \\
\hline BERTSUM & 38.81 & 16.50 & 31.27 \\
MATCHSUM & 24.86 & 4.66 & 18.41 \\
\hline PGNet & 29.70 & 9.21 & 23.24 \\
PGNet+Cov & 28.10 & 8.02 & 21.72 \\
BART & 45.14 & 22.27 & 37.25 \\
ProphetNet ${ }^{*}$ & - & - & - \\
PEGASUS & $\mathbf{4 7 . 2 1}$ & $\mathbf{2 4 . 5 6}$ & $\mathbf{3 9 . 2 5}$ \\
\hline ESACL & $\mathbf{4 4 . 6 4}$ & $\mathbf{2 1 . 6 2}$ & $\mathbf{3 6 . 7 3}$ \\
\hline * ProphetNet doesn't provide the result on XSUM.
\end{tabular}

Table 2: ROUGE (RG) evaluation on XSUM dataset

many baseline models and the comparable performance to the best-performed SOTA model with much smaller architecture. Comparing to BART and PEGASUS, our model has less trainable parameters: we have a 12-layer encoder and 6-layer decoder, which is much smaller than the architecture of BART: 12-layer encoder and 12-layer decoder, and the architecture of PEGASUS: 16-layer encoder and 16-layer decoder.

\subsection{Human Evaluation}

To further examine the quality of the generated summaries by ESACL, we conduct the human evaluation. Two common indicators in the literature, informativeness and fluency are used to measure the quality of summary (Huang et al., 2020; Xu et al., 2020). Informativeness measures whether the summary covers the important information from the input article and fluency focuses on if the generated summary is grammatically correct. We randomly select 100 articles from the XSUM test set and hire 7 fluent English speakers as our annotators to rate summaries generated by distil-BART and ESACL. They are required to give a comparison between the two generated summaries that are presented anonymously. Table 3 reports the human evaluation results. Overall, we find that our model is capable of capturing the key information of a document and the global semantics, which can be further demonstrated by the two example generated summaries from ESACL in Table 4.

\begin{tabular}{l|ccc}
\hline & Win & Tie & Loss \\
\hline Informativeness & $38.5 \%$ & $24.7 \%$ & $36.8 \%$ \\
Fluency & $19.5 \%$ & $61.0 \%$ & $19.5 \%$ \\
\hline
\end{tabular}

Table 3: Human evaluation results on XSUM dataset. 


\begin{tabular}{l|l}
\hline \multicolumn{1}{c|}{ Source article (abbreviated) } & \multicolumn{1}{c}{ Summary by ESACL } \\
\hline $\begin{array}{l}\text { The London trio are up for best UK act and best album, as } \\
\text { well as getting two nominations in the best song category. "We }\end{array}$ & $\begin{array}{l}\text { N-Dubz have revealed they were sur- } \\
\text { prised to be nominated for four Mobo } \\
\text { got told like this morning 'Oh I think you're nominated'", said } \\
\text { Dappy. "And I was like 'Oh yeah, which one?' And now we've } \\
\text { got nominated for four awards. I mean, wow!" ... }\end{array}$ \\
\hline $\begin{array}{l}\text { Since late November, Scotland's five mountain resorts have } \\
\text { attracted } 373,782 \text { customers. The ski season is estimated to } \\
\text { have attracted } £ 37.5 m \text { into the local economy. With fresh snow } \\
\text { on the slopes, CairnGorm Mountain expects skiing during the } \\
\text { first weekend of June. Recent figures from Ski Scotland showed } \\
\text { that this season's figures were better than the last bumper season } \\
\text { of } 2000-2001 . . .\end{array}$ & $\begin{array}{l}\text { A record number of skiers and snow- } \\
\text { boarders have visited Scotland's five }\end{array}$ \\
\hline
\end{tabular}

Table 4: Two example summaries by ESACL on XSUM dataset.

\section{Discussion}

\subsection{Impact of Contrastive Learning Component}

Since our model is warmed up using distil-BART, one could assume that the original distil-BART may simply need to be fine-tuned longer to achieve the same experimental results. Inspired by Peinelt et al. (2020), we perform an additional experiment to finetune distil-BART using the same experimental settings. By analyzing the results in Table 5, we can conclude that longer finetuning does not considerably boost distil-BART's performance.

\begin{tabular}{lccc}
\hline \multicolumn{1}{c}{ Model } & RG-1 & RG-2 & RG-L \\
\hline distil-BART & 41.23 & 19.38 & 38.11 \\
ESACL & $\mathbf{4 4 . 2 4}$ & $\mathbf{2 1 . 0 6}$ & $\mathbf{4 1 . 2 0}$ \\
\hline
\end{tabular}

(a) Performance on CNN/DM

\begin{tabular}{lccc}
\hline \multicolumn{1}{c}{ Model } & RG-1 & RG-2 & RG-L \\
\hline distil-BART & 44.41 & 21.40 & 36.50 \\
ESACL & $\mathbf{4 4 . 6 4}$ & $\mathbf{2 1 . 6 2}$ & $\mathbf{3 6 . 7 3}$ \\
\hline
\end{tabular}

(b) Performance on XSUM

Table 5: Finetune distil-BART under the same setting.

\subsection{Robustness Check}

We perform a robustness check for ESACL to better understand the impacts of different datasets on the constrastive learning performance. Follow Goel et al. (2021), we use several heuristics from literature to identify sub-populations of datasets. We first select top $10 \%$ and bottom $10 \%$ examples in the test set as two subpopulations based

\begin{tabular}{llcc}
\hline \multicolumn{2}{c}{ Metric } & Baseline & ESACL \\
\hline \multirow{2}{*}{ Length } & Longest & 15.89 & 15.96 \\
& Shortest & 23.35 & 23.14 \\
\hline \multirow{2}{*}{ Abstractive } & Most & 19.44 & 19.77 \\
& Least & 24.21 & 24.13 \\
\hline \multirow{2}{*}{ Distilled } & Most & 15.71 & 16.15 \\
& Least & 24.21 & 24.13 \\
\hline \multirow{2}{*}{ Position } & Latest & 17.30 & 17.60 \\
& Earliest & 22.22 & 22.40 \\
\hline
\end{tabular}

Table 6: Robustness Check on sub-populations defined by metrics using ROUGE-2. Baseline refers to distilBART.

on four metrics from Goel et al. (2021): length, abstractiveness, distillation and position. Then we evaluate the performance using ROUGE score on each subpopulation. Table 6 shows the performance of ESACL in each population comparing to distil-BART ${ }^{5}$. For length, we find that ESACL performs better than the baseline on the longest set, which is the hardest to summarize considering a large amount of information. For the most abstractive set, ESACL is more capable to reconstruct the text and achieves higher performance. This is more significant on the most distilled set, ESACL performs much better than the baseline by improving the performance by $2.8 \%$. We can also identify a smaller performance gap between two sub-populations for ESACL, thereby emphasizing that ESACL performs more robustly than the baseline.

\footnotetext{
${ }^{5}$ We report ROUGE-2 score in Table 6 . We include the detailed results for other ROUGE scores in Appendix B.
} 


\subsection{Ablation Study}

To better understand the contribution of different modules in ESACL to the performance, we conduct an ablation study using the XSUM dataset.

Document augmentation. As we illustrated the importance of data augmentation in contrastive learning (see Section 4.1), we design several document augmentations but we have not explored their impact on the summarization performance. Table 7 shows the result of ESACL using different augmentation methods ${ }^{6}$. We can clearly see that (1) the performance with different combinations of augmentation in abstractive text summarization does not vary too much. (2) The augmentation method that interrupts the document structure, such as document rotation (DR), is usually harmful to the performance, since the structure of the input document plays an important role.

\begin{tabular}{c|c|c|c|c}
\hline & RI & RD & RS & DR \\
\hline RI & 21.62 & 21.56 & 21.51 & 21.47 \\
RD & - & 21.59 & 21.58 & 21.38 \\
RS & - & - & 21.46 & 21.41 \\
DR & - & - & - & 21.11 \\
\hline
\end{tabular}

Table 7: Performance on XSUM dataset under different combinations of document augmentation.

Number of augmentation operations. One question we have not answered is what is the optimal number of augmentation operations. We expect this number to be in a reasonable range: too large can completely change the document's structure and too small does not add enough noise. So we design the experiment with varied number of sentences modified in the document augmentation and Table 8 shows the performance of ESACL under Random Deletion (RD) and Random Swap (RS) with different numbers of augmentation operations $n$. Given there are 19.77 sentences per article for XSUM on average (Narayan et al., 2018), we decide to choose $n$ from $[1,3,5]$. As we expected, both $n=5$ and $n=1$ performs worse than $n=3$. A reasonable choice for $n$ should be based on the characteristics of datasets under the guidance that data augmentation is useful to add some noises while preserving the critical information.

Layer freezing in the encoder. In all the above experiments, we need to freeze some layers in the

\footnotetext{
${ }^{6}$ we use ROUGE- 2 as the evaluation metrics, and we also report the results using ROUGE-L in Appendix C.
}

\begin{tabular}{l|ccc}
\hline & RG-1 & RG-2 & RG-L \\
\hline$n=1$ & 44.48 & 21.54 & 36.64 \\
$n=3$ & 44.52 & 21.58 & 36.59 \\
$n=5$ & 44.36 & 21.48 & 36.52 \\
\hline
\end{tabular}

Table 8: Performance on XSUM dataset with different numbers of augmentation operations.

encoder because of the memory limitation. Especially for contrastive learning, it benefits from the larger batch size comparing to supervised learning (Chen et al., 2020). This brings us to a trade-off between the batch size and the number of finetuned layers. Previous studies find that higher-level layers capture context-dependent aspects of text meaning while lower-level states model aspects of syntax (Peters et al., 2018; Mou et al., 2016). Thus, in our study, we freeze the first several $l$ layers of the encoder in ESACL. Table 9 reports the performance for different l's under Random Deletion (RD) and Random Swap (RS). When $l=12$, the model is fine-tuned only using the augmented documents, which makes contrastive learning ineffective. When compared to $l=6$, we clearly see the benefit of incorporating contrastive learning into the seq2seq during fine-tuning.

\begin{tabular}{l|ccc}
\hline & RG-1 & RG-2 & RG-L \\
\hline$l=6$ & 44.52 & 21.58 & 36.59 \\
$l=9$ & 44.41 & 21.47 & 36.55 \\
$l=12$ & 44.27 & 21.41 & 36.46 \\
\hline
\end{tabular}

Table 9: Performance on XSUM dataset when freezing the first $l$ layers in the encoder.

\section{Conclusion}

In this paper, we propose ESACL, an enhanced sequence-to-sequence model via contrastive learning to improve the performance of abstractive text summarization, where two critical components are jointly learned via fine-tuning. With several proposed sentence-level document augmentation, ESACL can build an autoencoder with a denoising capability through fine-tuning. We empirically evaluate ESACL on two datasets both quantitatively and qualitatively. The results demonstrate that ESACL outperforms several cutting-edge benchmarks. We also examine the impact of different augmentation strategies on the performance and explore the robustness of ESACL. 


\section{References}

Yonatan Belinkov and Yonatan Bisk. 2018. Synthetic and natural noise both break neural machine translation. In International Conference on Learning Representations.

Ting Chen, Simon Kornblith, Mohammad Norouzi, and Geoffrey Hinton. 2020. A simple framework for contrastive learning of visual representations. In International conference on machine learning, pages 1597-1607. PMLR.

Sumit Chopra, Michael Auli, and Alexander M Rush. 2016. Abstractive sentence summarization with attentive recurrent neural networks. In Proceedings of the 2016 Conference of the North American Chapter of the Association for Computational Linguistics: Human Language Technologies, pages 93-98.

Jacob Devlin, Ming-Wei Chang, Kenton Lee, and Kristina Toutanova. 2019. Bert: Pre-training of deep bidirectional transformers for language understanding. In Proceedings of the 2019 Conference of the North American Chapter of the Association for Computational Linguistics: Human Language Technologies, Volume 1 (Long and Short Papers), pages 4171-4186.

Hongchao Fang and Pengtao Xie. 2020. Cert: Contrastive self-supervised learning for language understanding. arXiv preprint arXiv:2005.12766.

Sebastian Gehrmann, Yuntian Deng, and Alexander Rush. 2018. Bottom-up abstractive summarization. In Proceedings of the 2018 Conference on Empirical Methods in Natural Language Processing, pages 4098-4109, Brussels, Belgium. Association for Computational Linguistics.

Karan Goel, Nazneen Rajani, Jesse Vig, Samson Tan, Jason Wu, Stephan Zheng, Caiming Xiong, Mohit Bansal, and Christopher Ré. 2021. Robustness gym: Unifying the nlp evaluation landscape.

Beliz Gunel, Jingfei Du, Alexis Conneau, and Ves Stoyanov. 2020. Supervised contrastive learning for pretrained language model fine-tuning. arXiv preprint arXiv:2011.01403.

Kaiming He, Haoqi Fan, Yuxin Wu, Saining Xie, and Ross Girshick. 2020. Momentum contrast for unsupervised visual representation learning. In Proceedings of the IEEE/CVF Conference on Computer Vision and Pattern Recognition, pages 9729-9738.

Dan Hendrycks, Steven Basart, Norman Mu, Saurav Kadavath, Frank Wang, Evan Dorundo, Rahul Desai, Tyler Zhu, Samyak Parajuli, Mike Guo, et al. 2020. The many faces of robustness: A critical analysis of out-of-distribution generalization. arXiv preprint arXiv:2006.16241.

Karl Moritz Hermann, Tomas Kocisky, Edward Grefenstette, Lasse Espeholt, Will Kay, Mustafa Suleyman, and Phil Blunsom. 2015. Teaching machines to read and comprehend. Advances in neural information processing systems, 28:1693-1701.

Luyang Huang, Lingfei Wu, and Lu Wang. 2020. Knowledge graph-augmented abstractive summarization with semantic-driven cloze reward. In Proceedings of the 58th Annual Meeting of the Association for Computational Linguistics, pages 50945107, Online. Association for Computational Linguistics.

Robin Jia and Percy Liang. 2017. Adversarial examples for evaluating reading comprehension systems. In Proceedings of the 2017 Conference on Empirical Methods in Natural Language Processing, pages 2021-2031.

Daniel Jurafsky and James H. Martin. 2020. Speech \& language processing.

Prannay Khosla, Piotr Teterwak, Chen Wang, Aaron Sarna, Yonglong Tian, Phillip Isola, Aaron Maschinot, Ce Liu, and Dilip Krishnan. 2020. Supervised contrastive learning. Advances in Neural Information Processing Systems, 33.

Oleksandr Kolomiyets, Steven Bethard, and MarieFrancine Moens. 2011. Model-portability experiments for textual temporal analysis. In Proceedings of the 49th annual meeting of the association for computational linguistics: human language technologies, volume 2, pages 271-276. ACL; East Stroudsburg, PA.

Mike Lewis, Marjan Ghazvininejad, Gargi Ghosh, Armen Aghajanyan, Sida Wang, and Luke Zettlemoyer. 2020a. Pre-training via paraphrasing. Advances in Neural Information Processing Systems, 33.

Mike Lewis, Yinhan Liu, Naman Goyal, Marjan Ghazvininejad, Abdelrahman Mohamed, Omer Levy, Veselin Stoyanov, and Luke Zettlemoyer. 2020b. Bart: Denoising sequence-to-sequence pretraining for natural language generation, translation, and comprehension. In Proceedings of the 58th Annual Meeting of the Association for Computational Linguistics, pages 7871-7880.

Bohan Li, Hao Zhou, Junxian He, Mingxuan Wang, Yiming Yang, and Lei Li. 2020a. On the sentence embeddings from pre-trained language models. arXiv preprint arXiv:2011.05864.

Tian Li, Xiang Chen, Shanghang Zhang, Zhen Dong, and Kurt Keutzer. 2020b. Cross-domain sentiment classification with in-domain contrastive learning. arXiv preprint arXiv:2012.02943.

Chin-Yew Lin. 2004. Rouge: A package for automatic evaluation of summaries. In Text summarization branches out, pages 74-81.

Yang Liu and Mirella Lapata. 2019. Text summarization with pretrained encoders. In Proceedings of the 2019 Conference on Empirical Methods in Natural Language Processing and the 9th International 
Joint Conference on Natural Language Processing (EMNLP-IJCNLP), pages 3721-3731.

Yinhan Liu, Myle Ott, Naman Goyal, Jingfei Du, Mandar Joshi, Danqi Chen, Omer Levy, Mike Lewis, Luke Zettlemoyer, and Veselin Stoyanov. 2019. Roberta: A robustly optimized bert pretraining approach. arXiv preprint arXiv:1907.11692.

Tom McCoy, Ellie Pavlick, and Tal Linzen. 2019. Right for the wrong reasons: Diagnosing syntactic heuristics in natural language inference. In Proceedings of the 57th Annual Meeting of the Association for Computational Linguistics, pages 3428-3448.

Lili Mou, Zhao Meng, Rui Yan, Ge Li, Yan Xu, Lu Zhang, and Zhi Jin. 2016. How transferable are neural networks in NLP applications? In Proceedings of the 2016 Conference on Empirical Methods in Natural Language Processing, pages 479-489, Austin, Texas. Association for Computational Linguistics.

Ramesh Nallapati, Feifei Zhai, and Bowen Zhou. 2017. Summarunner: A recurrent neural network based sequence model for extractive summarization of documents. In Proceedings of the AAAI Conference on Artificial Intelligence, volume 31.

Ramesh Nallapati, Bowen Zhou, Cicero Nogueira dos santos, Caglar Gulcehre, and Bing Xiang. 2016. Abstractive text summarization using sequence-tosequence RNNs and beyond. In Proceedings of The 20th SIGNLL Conference on Computational Natural Language Learning, pages 280-290, Berlin, Germany. Association for Computational Linguistics.

Shashi Narayan, Shay B Cohen, and Mirella Lapata. 2018. Don't give me the details, just the summary! topic-aware convolutional neural networks for extreme summarization. In Proceedings of the 2018 Conference on Empirical Methods in Natural Language Processing, pages 1797-1807.

Nicole Peinelt, Dong Nguyen, and Maria Liakata. 2020. tbert: Topic models and bert joining forces for semantic similarity detection. In Proceedings of the 58th Annual Meeting of the Association for Computational Linguistics, pages 7047-7055.

Matthew Peters, Mark Neumann, Mohit Iyyer, Matt Gardner, Christopher Clark, Kenton Lee, and Luke Zettlemoyer. 2018. Deep contextualized word representations. In Proceedings of the 2018 Conference of the North American Chapter of the Association for Computational Linguistics: Human Language Technologies, Volume 1 (Long Papers), pages 22272237.

Weizhen Qi, Yu Yan, Yeyun Gong, Dayiheng Liu, Nan Duan, Jiusheng Chen, Ruofei Zhang, and Ming Zhou. 2020. Prophetnet: Predicting future n-gram for sequence-to-sequence pre-training. In Proceedings of the 2020 Conference on Empirical Methods in Natural Language Processing: Findings, pages 2401-2410.
Siyuan Qiu, Binxia Xu, Jie Zhang, Yafang Wang, Xiaoyu Shen, Gerard de Melo, Chong Long, and Xiaolong Li. 2020. Easyaug: An automatic textual data augmentation platform for classification tasks. In Companion Proceedings of the Web Conference 2020, pages 249-252.

Alec Radford, Karthik Narasimhan, Tim Salimans, and Ilya Sutskever. Improving language understanding by generative pre-training.

Alec Radford, Jeffrey Wu, Rewon Child, David Luan, Dario Amodei, and Ilya Sutskever. 2019. Language models are unsupervised multitask learners. OpenAI blog, 1(8):9.

Alexander M Rush, Sumit Chopra, and Jason Weston. 2015. A neural attention model for abstractive sentence summarization. In Proceedings of the 2015 Conference on Empirical Methods in Natural Language Processing, pages 379-389.

Abigail See, Peter J Liu, and Christopher D Manning. 2017. Get to the point: Summarization with pointergenerator networks. In Proceedings of the 55th Annual Meeting of the Association for Computational Linguistics (Volume 1: Long Papers), pages 10731083.

Rico Sennrich, Barry Haddow, and Alexandra Birch. 2016. Improving neural machine translation models with monolingual data. In Proceedings of the 54th Annual Meeting of the Association for Computational Linguistics (Volume 1: Long Papers), pages 86-96.

Haoxiang Shi, Cen Wang, and Tetsuya Sakai. 2020. Self-supervised document clustering based on bert with data augment. arXiv preprint arXiv:2011.08523.

Kaitao Song, Xu Tan, Tao Qin, Jianfeng Lu, and TieYan Liu. 2019. Mass: Masked sequence to sequence pre-training for language generation. In International Conference on Machine Learning, pages 5926-5936. PMLR.

Jiwei Tan, Xiaojun Wan, and Jianguo Xiao. 2017. Abstractive document summarization with a graphbased attentional neural model. In Proceedings of the 55th Annual Meeting of the Association for Computational Linguistics (Volume 1: Long Papers), pages 1171-1181.

Ashish Vaswani, Noam Shazeer, Niki Parmar, Jakob Uszkoreit, Llion Jones, Aidan N Gomez, Łukasz Kaiser, and Illia Polosukhin. 2017. Attention is all you need. In Proceedings of the 31st International Conference on Neural Information Processing Systems, pages 6000-6010.

William Yang Wang and Diyi Yang. 2015. That's so annoying!!!: A lexical and frame-semantic embedding based data augmentation approach to automatic categorization of annoying behaviors using\# petpeeve tweets. In Proceedings of the 2015 conference on 
empirical methods in natural language processing, pages 2557-2563.

Sebastien C Wong, Adam Gatt, Victor Stamatescu, and Mark D McDonnell. 2016. Understanding data augmentation for classification: when to warp? In 2016 international conference on digital image computing: techniques and applications (DICTA), pages 16. IEEE.

Song Xu, Haoran Li, Peng Yuan, Youzheng Wu, Xiaodong He, and Bowen Zhou. 2020. Self-attention guided copy mechanism for abstractive summarization. In Proceedings of the 58th Annual Meeting of the Association for Computational Linguistics, pages 1355-1362, Online. Association for Computational Linguistics.

Adams Wei Yu, David Dohan, Minh-Thang Luong, Rui Zhao, Kai Chen, Mohammad Norouzi, and Quoc V Le. 2018. Qanet: Combining local convolution with global self-attention for reading comprehension. arXiv preprint arXiv:1804.09541.

Jingqing Zhang, Yao Zhao, Mohammad Saleh, and Peter Liu. 2020. Pegasus: Pre-training with extracted gap-sentences for abstractive summarization. In International Conference on Machine Learning, pages 11328-11339. PMLR.

Xiang Zhang, Junbo Zhao, and Yann LeCun. 2015. Character-level convolutional networks for text classification. In Advances in neural information processing systems, pages 649-657.

Ming Zhong, Pengfei Liu, Yiran Chen, Danqing Wang, Xipeng Qiu, and Xuanjing Huang. 2020. Extractive summarization as text matching. In Proceedings of the 58th Annual Meeting of the Association for Computational Linguistics, pages 6197-6208, Online. Association for Computational Linguistics.

Qingyu Zhou, Nan Yang, Furu Wei, and Ming Zhou. 2017. Selective encoding for abstractive sentence summarization. In Proceedings of the 55th Annual Meeting of the Association for Computational Linguistics (Volume 1: Long Papers), pages 10951104.

Junnan Zhu, Qian Wang, Yining Wang, Yu Zhou, Jiajun Zhang, Shaonan Wang, and Chengqing Zong. 2019. Ncls: Neural cross-lingual summarization. In Proceedings of the 2019 Conference on Empirical Methods in Natural Language Processing and the 9th International Joint Conference on Natural Language Processing (EMNLP-IJCNLP), pages 3045-3055. 


\section{A Implementation}

Our implementation in this paper is warmed up using the pre-trained model from HuggingFace: for CNN/DM, we use "sshleifer/distilbart-cnn-12-6"

${ }^{7}$. For XSUM: we use "sshleifer/distilbart-xsum$12-6 "{ }^{8}$. These two models are fine-tuned on the corresponding datasets. Comparing to the original BART, distil-BART contains much less parameters, which is also able to achieve comparable performance.

For CNN/DM, we also add the post-processing step after the generation, which is common in literature. We use the same post-processing program ${ }^{9}$ from SOTA model ProphetNet (Qi et al., 2020).

\section{B Robustness Check}

For robustness check, we report the performance using ROUGE-1, ROUGE-2 and ROUGE-L in Figure 3 . We can have the same conclusion by analyzing the numbers: ESACL is more capable to perform well and robust for documents with different features, since ESACL can perform better than the baseline in the subpopulation which is more challenging to summarize. For example, for the most distilled subpopulation which requires the language model to reconstruct a lot of information from the document (refer to "Most Distilled"), ESACL performs better than the baseline by $1.6 \%, 2.8 \%$ and $1.4 \%$ in RG-1, RG-2 and RG-L. We can also find the performance gap for each subpopulation from ESACL is smaller. The key conclusion from the robustness check is ESACL performs more stable and robust on different types of the input document.

\section{Document Augmentation}

In Section 6.3, we perform an ablation study to analyze the impacts of choosing different document augmentation strategies on the model performance. Table 10 records the performance evaluated by ROUGE- 2 and ROUGE-L under different combinations of document augmentation methods. Numbers in parenthesis refer to the ROUGE-L score. We have the consistent conclusion with our previous analysis in Section 6.3.

\begin{tabular}{c|c|c|c|c}
\hline & RI & RD & RS & DR \\
\hline \multirow{2}{*}{ RI } & 21.62 & 21.56 & 21.51 & 21.47 \\
& $(36.73)$ & $(36.65)$ & $(36.62)$ & $(36.55)$ \\
RD & - & 21.59 & 21.58 & 21.38 \\
& - & $(36.65)$ & $(36.59)$ & $(36.38)$ \\
RS & - & - & 21.46 & 21.41 \\
& - & - & $(36.49)$ & $(36.44)$ \\
DR & - & - & - & 21.11 \\
& - & - & - & $(36.12)$ \\
\hline
\end{tabular}

Table 10: Performance on XSUM dataset under different combinations of document augmentation. We report the ROUGE-2 and ROUGE-L (in parenthesis) F1 score.

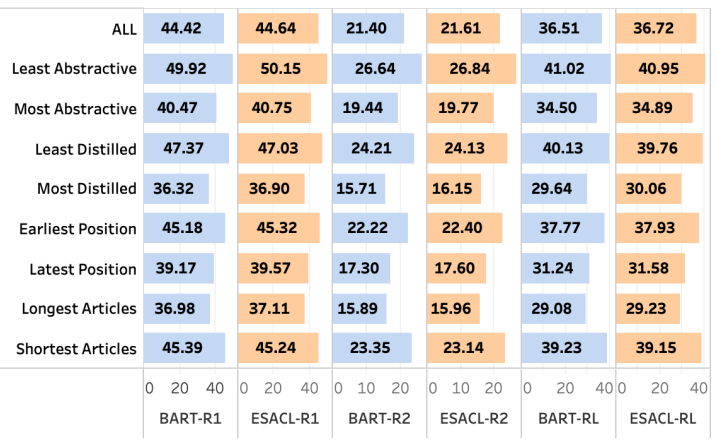

Figure 3: Robustness check on XSUM dataset.

\footnotetext{
${ }^{7}$ https://huggingface.co/sshleifer/distilbart-cnn-12-6

${ }^{8}$ https://huggingface.co/sshleifer/distilbart-xsum-12-6

${ }^{9}$ https://github.com/microsoft/ProphetNet
} 